\title{
Two-Photon Fluorescence Imaging Super-Enhanced by Multishell Nanophotonic Particles, with Application to Subcellular pH
}

\author{
Aniruddha Ray, Yong-Eun Koo Lee, Gwangseong Kim, and Raoul Kopelman*
}

\begin{abstract}
A novel nanophotonic method for enhancing the two-photon fluorescence signal of a fluorophore is presented. It utilizes the second harmonic (SH) of the exciting light generated by noble metal nanospheres in whose near-field the dye molecules are placed, to further enhance the dye's fluorescence signal in addition to the usual metalenhanced fluorescence phenomenon. This method enables demonstration, for the first time, of two-photon fluorescence enhancement inside a biological system, namely live cells. A multishell hydrogel nanoparticle containing a silver core, a protective citrate capping, which serves also as an excitation quenching inhibitor spacer, a pH indicator dye shell, and a polyacrylamide cladding are employed. Utilizing this technique, an enhancement of up to 20 times in the two-photon fluorescence of the indicator dye is observed. Although a significant portion of the enhanced fluorescence signal is due to one-photon processes accompanying the $\mathrm{SH}$ generation of the exciting light, this method preserves all the advantages of infrared-excited, two-photon microscopy: enhanced penetration depth, localized excitation, low photobleaching, low autofluorescence, and low cellular damage.
\end{abstract}

\section{Introduction}

Multiphoton fluorescence is a very powerful technique for spectroscopic measurements and for biomedical imaging in cells or tissues. Multiphoton excited fluorescence is a nonlinear optical process in which two or more photons, typically in the near-infrared (NIR) region, are absorbed simultaneously and the fluorescence occurs at a shorter wavelength (higher frequency), typically in the visible region. Twophoton fluorescence is the most commonly used option, due to its relatively higher absorption cross section than other multiphoton fluorescence modes. Multiphoton fluorescence has higher spatial resolution than one-photon fluorescence,

A. Ray, Prof. R. Kopelman BioPhysics, University of Michigan 930 N. University Ave. Ann Arbor, MI 48109, USA

E-mail: kopelman@umich.edu

Dr. Y.-E. K. Lee, Dr. G. Kim, Prof. R. Kopelman Department of Chemistry

University of Michigan

930 N. University Ave. Ann Arbor, MI 48109, USA

DOI: $10.1002 / \mathrm{smll} .201102664$ because the fluorescence's quadratic (two-photon) [or cubic (three-photon)] dependence on the excitation intensity turns it into a highly localized and spatially confined excitation. It also allows the use of NIR radiation for exciting fluorescent dyes in the visible range, thus enabling a much enhanced penetration depth, on the order of several millimeters, in tissues. Furthermore, it also significantly reduces both autofluorescence from, and photodamage to, the cells/tissues, and thus increases further the signal-to-noise ratio. ${ }^{[1-3]}$ However, one of the biggest disadvantages of using nonlinear excitation is its extremely low absorption/fluorescence cross section. The probability of simultaneously absorbing two low-energy photons is quite low. Thus, for most dyes, the two-photon fluorescence signal is much weaker, by many orders of magnitude, than the one-photon fluorescence signal. The weak nature of this two-photon fluorescence emanating from the dye molecules has severely limited in vivo applications, penetration depth, and choice of dye, thus preventing it from achieving its full potential.

Several methods have been utilized to enhance the twophoton fluorescence signal, ${ }^{[4-13]}$ some of which have indeed shown orders of magnitude enhancements in the two-photon fluorescence signal, and also enabled optical microscopy 
beyond the diffraction limit. Most of these near-field optics or nanophotonic techniques have used metallic films, nanoparticles, fractal nanoislands, or tips, which, however, would all be limited to the cellular membrane or tissue surface, and thus would not be suitable for intracellular or deep in vivo imaging. Also, some of the above techniques use total internal reflection fluorescence (TIRF) microscopy to couple the light into the surface plasmon resonance (SPR) modes. TIRF microscopy is a very sensitive technique that has a very high signal-to-noise ratio but, unfortunately, it is unable to exploit one of the main advantages of using NIR light-its large penetration depth. In TIRF microscopes the penetration depth is determined by the decay of the evanescent waves, which is typically only several hundred nanometers. One possible way to enhance the two-photon fluorescence, which would overcome the above limitations, was introduced using the technique of resonance energy transfer between semiconductor nanocrystals and probes; ${ }^{[14]}$ however, this technique has not been demonstrated in cells or tissues. So far, no technology has been established that can enhance two-photon fluorescence signals inside cells, or deep inside tissues.

The two-photon fluorescence enhancement in the previous studies is mostly based on SPR, also known as the metal-enhanced fluorescence (MEF) effect. The presence of metallic nanoparticles can either enhance or quench the fluorescence of a fluorophore adsorbed on its surface, with a delicate dependence on the distance from the metal surface ${ }^{[15-17]}$ and, presumably, the molecular orientation. The enhancement in fluorescence generally occurs when fluorophores are present close enough to the metal surface, due to the presence of the metal's surface plasmons (SPs), which lead to an enhanced local electric field near the metal surface. SPs are oscillating free electrons on the surface of the metal. If the dye molecules are too close to the metal surface $(0-5 \mathrm{~nm})$, their fluorescence is quenched, because the excitation fluorescence energy is transferred to the metal surface and is dissipated as heat. ${ }^{[15]}$ For larger separations there is an increase in fluorescence, either due to an enhancement of the incident light field, especially around the edges of the nanoparticles, or due to an increase in the radiative decay rate. ${ }^{[16,17]}$ Apart from SPR, Förster resonance energy transfer (FRET) has also been utilized to enhance the two-photon fluorescence from fluorophores. ${ }^{[14]}$ The fluorescence is enhanced due to a direct resonance energy transfer from the oscillating electrons of the donor to the fluorophore, due to a transitiondipole-transition-dipole coupling.

Herein, we present a new strategy to enhance two-photon fluorescence in parallel with the MEF effect, by exploiting the hyper-Rayleigh scattering (HRS) of the excitation light in the presence of metal nanoparticles, as shown in Figure 1. This is the first demonstration of such fluorescence enhancement inside living cells. HRS is a second-order nonlinear process that leads to incoherent second harmonic generation (SHG), that is, production of light at half the wavelength of the excitation laser. The phenomenon of HRS arises from the induceddipole polarization and the quadrupole polarization, due to the surface-induced nonlinear susceptibility. The quadrupole contribution is more significant for nanoparticles greater than $30 \mathrm{~nm} \cdot{ }^{[18]}$ Metal nanoparticles such as gold or silver have been used for HRS due to their large hyperpolarizability $(\beta)$, which gives rise to very large second harmonic signals. ${ }^{[19-23]}$ HRS has been used for certain biological applications, such as the detection of the Tau protein, identification of Escherichia coli bacteria, sequence-specific HCV RNA quantification etc., by looking at the change in the scattering intensity of the second harmonic signal. ${ }^{[24-26]}$ However, this technique has not been used so far to enhance multiphoton fluorescence. For two-photon fluorescence, the excitation wavelength used is twice the wavelength of the normal (single-photon) excitation of the fluorescent dye. By combining the dyes with metal nanoparticles, the second harmonic of the same excitation light frequency used for the two-photon fluorescence can be used to excite the fluorescent dye. This second harmonic is generated by the metal nanoparticles. Since the one-photon fluorescence cross section is orders of magnitude larger than the two-photon fluorescence cross section, this technique has the potential to bring about a significant enhancement in the fluorescence of the indicator dyes, compared to normal twophoton fluorescence.

Although a significant contribution of the fluorescence signal is due to single-photon excitation, this nanophotonic technique still exploits all the advantages of NIR-excited multiphoton fluorescence, such as reduced scattering, larger penetration depth, highly localized excitation, low autofluorescence, and high signal-to-noise ratio. The technique can be applied using any type of metal nanoparticle that has a large hyperpolarizability value, such as gold or silver. We note that metal core/polymer shell type nanoparticles ${ }^{[27-29]}$ or gold nanoparticles ${ }^{[30]}$ have already been used to enhance 
the one-photon fluorescence signal ${ }^{[27,28]}$ and second harmonic signal ${ }^{[29,30]}$ but not yet for two-photon fluorescence.

To demonstrate the in vivo applicability of this technique, we prepared a core/shell nanoparticle $\mathrm{pH}$ sensor by encapsulating into an inert polyacrylamide matrix a $\mathrm{pH}$-sensitive dye, 8-hydroxypyrene-1,3,6-trisulfonic acid (HPTS), adsorbed onto citrate-coated silver nanospheres. The citrate coating helps maintain some distance between the dye molecules and the metal surface. The polyacrylamide matrix shell of our nanoparticles has several chemical and biological advantages, as reported previously, ${ }^{[31-34]}$ in addition to obviating metal nanoparticle clusterization. We observed a maximum enhancement of 20 times for the fluorescence intensity of HPTS, when irradiated with focused femtosecond pulses at a selected NIR wavelength, compared to free dye or nanoparticles containing dye only. We applied this nanoparticle sensor to intracellular $\mathrm{pH}$ sensing, which demonstrates the feasibility of these nanosensors for imaging applications in cells and tissues, and for future in vivo sensing. We note that nanoparticle-based fluorescence sensors, called PEBBLEs (photonic explorers for bioanalysis with biologically localized embedding), which have been demonstrated to have numerous advantages over fluorescent molecular probes, such as better sensitivity, selectivity, and targetability, ${ }^{[31-34]}$ can be seamlessly combined with this new multishell approach for enhancing two-photon biochemical imaging.

\section{Results and Discussion}

\subsection{Characterization of Nanophotonic Particles}

We confirmed the presence of HRS by irradiating the silver nanoparticles, without the dye and polymer coating, with a high-intensity femtosecond laser and collecting the second harmonic generated signal using a photomultiplier tube (PMT). Figure 2a shows the second harmonic spectrum generated by the $60 \mathrm{~nm}$ silver nanoparticles, when irradiated by the $900 \mathrm{~nm}$ laser line. Under biologically relevant laser intensity conditions we see a weak second harmonic signal generated by the $60 \mathrm{~nm}$ silver nanospheres, which is quite easily distinguishable from the background. However, we did not see second harmonic signals generated from $10 \mathrm{~nm}$ silver nanospheres. This is due to the much lower SHG, possibly because of the low $\beta$ (hyperpolarizability) value of the $10 \mathrm{~nm}$ spheres relative to the $60 \mathrm{~nm}$ ones.

To utilize the HRS, that is, the second harmonic signal generated by the silver nanoparticles, to enhance the fluorescence intensity, we prepared nanosensors by encapsulating dye-adsorbed silver nanoparticles (10 or $60 \mathrm{~nm}$ ) into a polyacrylamide matrix. Encapsulating the silver in the polyacrylamide matrix prevents the nanoparticles from aggregating, which is one of the main challenges with metallic nanoparticles. The small pore size of the matrix prevents not only the silver but also the dye molecules from interacting with the proteins or other macromolecular components present inside the cell compartments. However, the hydrogel pores allow free flow of small ions and molecules, such as $\mathrm{H}^{+}, \mathrm{Ca}^{2+}, \mathrm{K}^{+}$, or $\mathrm{O}_{2}$, thus enabling the nanoparticle core to act as a chemical sensor. ${ }^{[31-34]}$ The prepared nanoparticle sensors show $\mathrm{pH}$-dependent two-photon fluorescence excitation spectra, as presented in Figure $2 \mathrm{~b}$. The inductively coupled plasma (ICP) measurements show a high amount of silver encapsulation inside the nanoparticles. This is also confirmed by looking at the powder X-ray diffraction (XRD) pattern, as shown in Figure 2c. The size of the nanoparticles containing a silver core varies from 90 to $130 \mathrm{~nm}$, according to the transmission electron microscopy (TEM) images. A typical TEM image of a nanoparticle containing silver is shown in Figure 2d. The surface charge of the nanoparticles was measured to be about $+18 \mathrm{mV}$.

We confirmed the absence of silver nanosphere clusters in our nanoparticles by looking at the bulk absorption spectra of these nanoparticles. Free silver nanospheres, when clustered, have a second absorption peak in the range 600-700 nm. But for our nanoparticles we do not observe any absorption band at 600-700 $\mathrm{nm}$. This bulk absorption study further confirms that most, if not all, of the embedding polyacrylamide nanoparticles contain a single silver nanosphere and not a cluster of nanospheres. From our fluorescence and 
absorbance measurements we find that the concentration of dye in the nanoparticles with $60 \mathrm{~nm}$ core was about $1.1 \%$ by weight, while the $10 \mathrm{~nm}$ core nanoparticles had only $0.57 \%$ dye (by weight) encapsulated in them. The nanosensors are highly soluble in water and phosphate-buffered saline (PBS). The dye leaching from the nanoparticles was extremely low. Generally, dye leaching is very significant when the dye is just encapsulated and not covalently linked to the matrix. With the silver core the dye leaching from the nanoparticles was about $0.2 \%$, under constant stirring in water for $24 \mathrm{~h}$, which is 50 times less than for nanoparticles without a silver core. ${ }^{[34]}$ This reduction in dye leaching is because the dye gets adsorbed onto the surface of the silver cores. This low degree of leaching out is quite remarkable, and comparable to the low leaching of dyes that are covalently linked to the matrix. We tested the toxicity of the nanoparticles by performing MTT assays. These toxicity assays show that the nanoparticles are practically nontoxic to cells, after $4 \mathrm{~h}$ of incubation at a $1 \mathrm{mg} \mathrm{mL}^{-1}$ concentration, and exhibit a higher than $98 \%$ cell viability.

\subsection{Two-Photon Fluorescence Enhancement}

The enhancement of the two-photon signal was observed by comparing the fluorescence signal from the nanoparticles with a silver core to that from equivalent amounts of free dye as well as nanoparticles containing dye but no nanoparticle. HPTS has multiply peaked, broad absorption bands around 370,405 , and $450 \mathrm{~nm}$, and an emission maximum at $510 \mathrm{~nm}$. The nanoparticles were excited at different wavelengths from 740 to $920 \mathrm{~nm}$, and the fluorescence emission between 480 and $580 \mathrm{~nm}$ was measured. The wavelength-dependent enhancement of the fluorescence signal is shown in Figure 3. For the $60 \mathrm{~nm}$ silver core there is a maximum enhancement of about 20 times (Figure 3a) and for the $10 \mathrm{~nm}$ silver core the enhancement is only 2.2 times (Figure 3b). We also found a similar enhancement trend for the nanoparticles with a 60 or $10 \mathrm{~nm}$ silver core, by measuring the fluorescence intensity between 560 and $600 \mathrm{~nm}$, which was carried out to avoid any error due to possible interference at shorter wavelength, despite the much weaker fluorescence signal intensity.
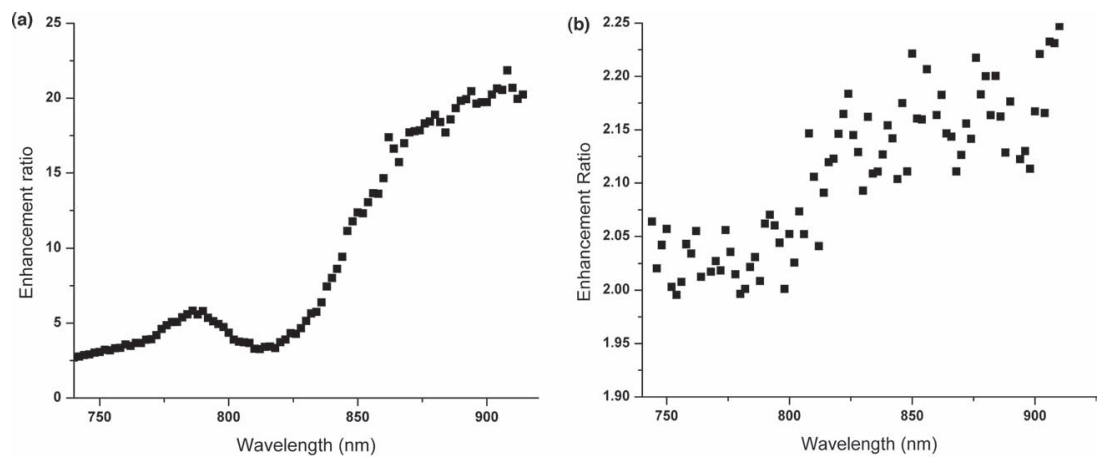

Figure 3. Two-photon fluorescence enhancement of the HPTS-nanoparticles with $60 \mathrm{~nm}$ silver core (a) and $10 \mathrm{~nm}$ silver core (b). For the $60 \mathrm{~nm}$ silver the maximum enhancement is 20 times whereas for the $10 \mathrm{~nm}$ silver the maximum enhancement is only 2.2 times.
We hypothesize that the major reason for the higher enhancement (about 10 times) in the fluorescence signal of the nanoparticles with the $60 \mathrm{~nm}$ silver cores-compared to the nanoparticles with $10 \mathrm{~nm}$ silver cores-may be the generation of second harmonic photons by the silver core, which in turn excites the fluorescent dye in the core's vicinity. This latter process is a one-photon fluorescence process and thus is much more efficient than the two-photon fluorescence, and has the potential to lead to a many-fold increase in the fluorescence signal. This hypothesis is mainly based on the wavelength-dependent enhancement trend, as well as on a comparison with a dye (rhodamine 6G) that does not have an overlapping absorption spectrum with the second harmonic of the exciting light. It has been shown previously that the hyperpolarizability $(\beta)$ of the silver nanoparticles increases with particle size, ${ }^{[19,26]}$ which is in accord with the difference in the fluorescence enhancement between the nanoparticles with a $60 \mathrm{~nm}$ silver core and those with a $10 \mathrm{~nm}$ core. Figure 3 shows that the enhancement of the fluorescence is about a factor of 3-5 from 740 to $820 \mathrm{~nm}$ and then, from $820 \mathrm{~nm}$, it starts to increase until the enhancement factor reaches a maximum value of 20 . The reduced enhancement at lower wavelengths is probably due to reabsorption of the second harmonic wavelength generated by the silver nanoparticles. Silver has a strong absorption around $400 \mathrm{~nm}$ and thus most of the second harmonic photons when excited at $800 \mathrm{~nm}$ are reabsorbed by the silver and thus cannot be used to excite the dye molecules. In contrast, from $900 \mathrm{~nm}$ onward excitation, the second harmonic generated is completely utilized to excite the dye molecules, while there is no absorption by the silver nanoparticles. In addition, silver has high reflectivity around $450 \mathrm{~nm}$, thus increasing the path length of the photons, around $450 \mathrm{~nm}$, inside the solution. This multireflection phenomenon has also been shown to enhance the fluorescence of dyes. ${ }^{[35]}$ We see this trend for both the 60 and $10 \mathrm{~nm}$ silver nanoparticles. We also compared nanoparticles containing different dye concentrations ranging from 0.5 to $1.1 \%$. However, no major difference in enhancement was observed.

The above enhancement method preserves all the advantages traditionally associated with two-photon fluorescence. The use of NIR radiation to generate the second harmonic ensures a large tissue penetration depth. Also, the "second harmonic" generated is highly localized, which is then absorbed only by the dye in the vicinity of the silver; thus we still get automatic image cross-sectioning, which is one of the biggest advantages of two-photon microscopy..$^{[1-3]}$ The localized excitation also minimizes photobleaching. The second harmonic generated is localized and relatively weak and thus does not cause autofluorescence from, or photodamage to, the cells.

To determine the contribution of the other MEF effects, such as SPR and resonance energy transfer, we tested silver nanoparticles with the dye rhodamine $6 \mathrm{G}$, to see the effect of SPR on the enhancement of the fluorescence generated by the dye molecules. Rhodamine and its 
derivatives (especially rhodamine $6 \mathrm{G}$ and rhodamine $\mathrm{B}$ ) are commonly used dyes that have been extensively utilized previously to study the MEF effects. ${ }^{[7,9,16,36]}$ The nanoparticles containing rhodamine dye were around $170 \mathrm{~nm}$ in size and contained $0.7 \%$ dye (by weight). We did not see much silverinduced enhancement of the fluorescence signal emanating from the rhodamine dye. We observed a maximum fluorescence signal enhancement of 1.4 and 2.8 times with the 10 and $60 \mathrm{~nm}$ silver spheres, respectively. Thus, SPR or FRET does not seem to be the major contributor to the overall enhancement observed with HPTS. Notably, the absorption spectrum of the rhodamine does not overlap with the second harmonic wavelength of the incident light, and thus enhancement due to HRS is excluded here. The comparison between the enhancement factor for HPTS and rhodamine is shown in Table 1.

One point of interest here is the distance and the interaction between the HPTS dye and the silver nanospheres. To get a rough estimate of the distance between the dye molecules and the silver surface, we looked at the one-photon fluorescence enhancement. Here, we observed a slight quenching of the fluorescence signal for the polyacrylamide-coated silvercontaining dye, as compared to the free dye at equal concentration. The fluorescence signal intensity of the nanoparticle was about $80 \%$ that of the free dye at $405 \mathrm{~nm}$. This indicates that the dye molecule is in very close proximity to the silver nanosphere surface, as for the quenching/enhancement theory described before; ${ }^{[15]}$ though the molecules may be in an altered orientation, they may not be quite "touching", which might have resulted in total quenching of the fluorescence (see more on this below). That the dye is physically adsorbed near the silver surface is also corroborated by the fact that we do not observe any dye leaching from the polyacrylamide nanoparticles embedded with silver in them, whereas without the silver there is as much as $8 \%$ dye leaching under similar conditions. ${ }^{[34]}$ Interestingly, total quenching is not observed. This may be explained in two ways: 1) the silver nanospheres employed have a citrate cap, so the capping citrate molecules may prevent the dye molecules from being totally quenched by helping to maintain a certain minimal distance between the metal and the dye; 2) the quenching may also depend on the orientation of the dye with respect to the metal surface, so if the orientation of the dye is nearly perpendicular to the silver surface then it might prevent it from being totally quenched, even though it is still in close proximity to that surface. This distance between the dye and the silver surface is not optimal for our enhancement studies and the two-photon enhancement obtained from our method could probably be much higher, upon optimization, than the factor of 20 that was observed. A quantitative study on this effect is planned.

Table 1. Comparison of fluorescence enhancement from HPTS and rhodamine-containing nanoparticles.

\begin{tabular}{lcccccc}
\hline Nanoparticle size & & \multicolumn{2}{c}{$60 \mathrm{~nm}$ silver } & & \multicolumn{2}{c}{$10 \mathrm{~nm}$ silver } \\
\cline { 1 - 3 } \cline { 5 - 6 } \cline { 5 - 6 } Mye & HPTS & Rhodamine 6G & & HPTS & Rhodamine 6G \\
\hline Maximum enhancement factor & 20 & 2.8 & & 2.2 & 1.4 \\
\hline
\end{tabular}

Importantly, although the close distance between the dye and the metal surface causes some loss of fluorescence signal, it still prevents the dye from leaching, which is also a very significant point. We plan to address this question of optimal distance, to prevent any fluorescence quenching yet to minimize the dye leaching, by using different techniques to control the distance between the dye and the silver, in a separate study. The lack of single-photon enhancement for the HPTS nanoparticles with silver core further demonstrates that the MEF effect is not so prominent. Thus, it does not play a major role in the two-photon fluorescence enhancement as well.

\subsection{Intracellular pH Mapping}

These nanoparticles were tested for $\mathrm{pH}$ sensing in cells. As most of the drug-delivery mechanisms depend on the $\mathrm{pH}$, $\mathrm{pH}$ is an extremely important physiological parameter. Many anticancer drugs are designed to release their contents at low $\mathrm{pH}$, and thus the knowledge of the exact $\mathrm{pH}$ inside the cells or tissues should be very helpful for drug design. The two-photon excitation spectra of the nanoparticles show a $\mathrm{pH}$-dependent peak at $900 \mathrm{~nm}$. It corresponds to the singlephoton absorption peaks at $450 \mathrm{~nm}$. The one-photon absorbance peaks of HPTS are highly $\mathrm{pH}$ sensitive. The absorption at $370 \mathrm{~nm}$ (between 350 and $410 \mathrm{~nm}$ ) increases at lower $\mathrm{pH}$, whereas the absorption at $450 \mathrm{~nm}$ (410 to $490 \mathrm{~nm}$ ) decreases at lower $\mathrm{pH}$. A dramatic $\mathrm{pH}$-dependent change is observed at $900 \mathrm{~nm}$, but, contrary to the one-photon $\mathrm{pH}$ response, we do not see much change elsewhere around $800 \mathrm{~nm}$, or for lower wavelengths. The fluorescence intensity ratio between the peaks of the two two-photon excitation wavelengths, at $900 \mathrm{~nm}$ and at an isosbestic point $(740 \mathrm{~nm})$, increases linearly over the $\mathrm{pH}$ range between 6.0 and 8.0 for these nanoparticle sensors. The $\mathrm{pH}$ calibration curve, based on the ratio of fluorescence intensities with excitations at 900 and $740 \mathrm{~nm}$, is shown in Figure 4. It is nearly linear, with an $R^{2}$ value of 0.98 . We also checked the response of the nanoparticles to ions such as $\mathrm{K}^{+}$and $\mathrm{Na}^{+}$, which are abundantly present in the cell. This was done by using various concentrations ( 0 to $0.5 \mathrm{M}$ ) of $\mathrm{KCl}$ and $\mathrm{NaCl}$ in a buffer solution. We did not observe any changes in the response of the nanoparticles to $\mathrm{K}^{+}$and $\mathrm{Na}^{+}$in these solutions.

MDA-MB-435 cancer cells were incubated with the nanoparticles for $3 \mathrm{~h}$, then washed and used for imaging. The nanoparticles were taken up by endocytosis. A two-photon fluorescence image of the live MDA cells containing the nanoparticles is shown in Figure 5. Using the ratio of the fluorescence intensity when excited at 900 and $740 \mathrm{~nm}$, an average intracellular $\mathrm{pH}$ value of $6.5 \pm 0.2$ is obtained using the $60 \mathrm{~nm}$ silver core particles. The low $\mathrm{pH}$ value measured is probably because these nanoparticles are trapped in acidic vesicles, such as late endosomes or lysosomes, which are acidic in nature. The absence of any targeting peptide on the nanoparticle surface precludes the involvement of receptor-mediated endocytosis in the cellular uptake of the nanoparticles. However, the highly positive surface charge of the nanoparticles ( $\approx+18 \mathrm{mV}$ by zeta potential measurement) confers their strong affinity for the negatively charged cellular membrane, 


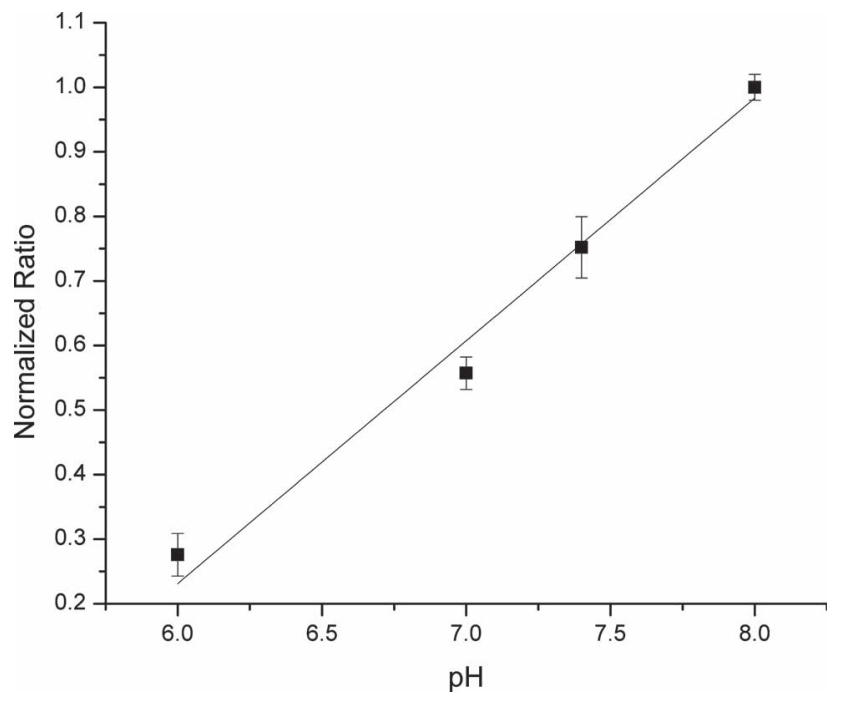

Figure 4. $\mathrm{pH}$ calibration curve of the nanoparticles. The curve was constructed by taking the ratio of the fluorescence when excited by 900 and $740 \mathrm{~nm}$ photons. $R^{2}=0.98$.

and promotes their uptake into the cell. Once endocytosed, the nanoparticles are destined for the lysosomes due to the absence of a targeting signal on their surface.

To check this preferential nanosensor trapping hypothesis, we looked at the co-localization of the nanoparticles with the acidic compartments, using Lysotracker. The Lysotracker probes specifically stain the acidic compartments of the cells and have been widely used to visualize lysosomes, which are strongly acidic, relative to other compartments of the cell. ${ }^{[34]}$ The fluorescence images were obtained by exciting

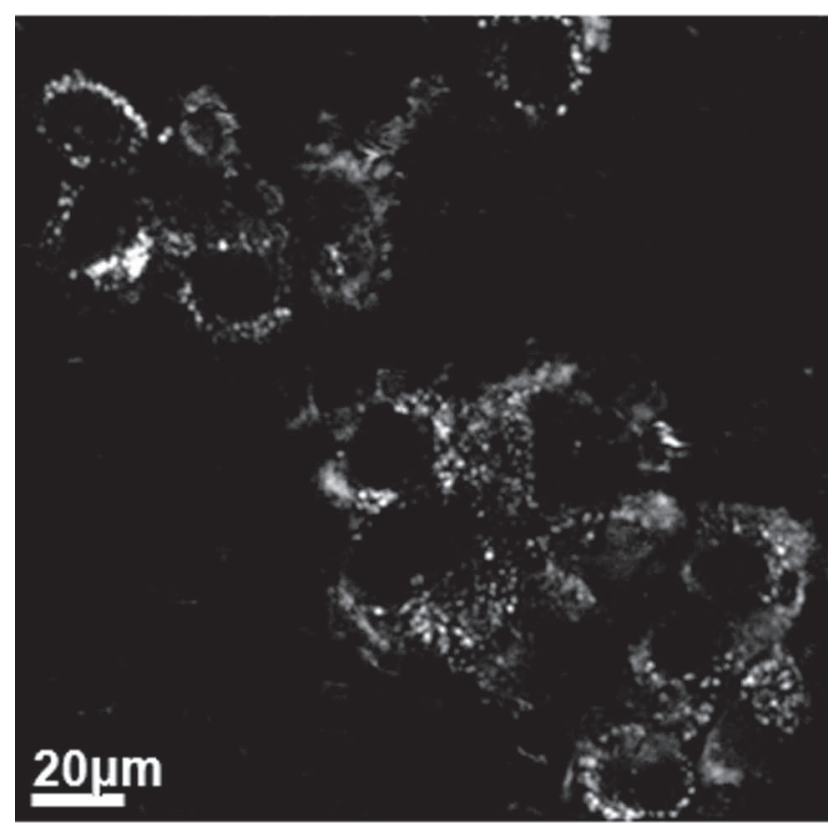

Figure 5. Two-photon fluorescence image of MDA-MB-435 cells with $\mathrm{pH}$-sensing core/shell nanoparticles, excited at $900 \mathrm{~nm}$; scale bar: $20 \mu \mathrm{m}$. Monitored is the $\mathrm{pH}$ of the acidic endosomes/lysosomes in which the nanosensors are preferentially localized/trapped, in contrast to other cell locations. the nanoparticles at 405 and $450 \mathrm{~nm}$, and the Lysotracker at $568 \mathrm{~nm}$, while collecting the fluorescence emission at 510 and $600 \mathrm{~nm}$, for the nanoparticle and Lysotracker dye, respectively. The overlaid image is shown in Figure 6. Significant co-localization of the nanoparticles with the acidic compartments is observed. A rough estimate shows that about $50 \%$ of the nanoparticles are in acidic compartments. This estimation was performed using the Image $\mathrm{J}$ software and by comparing the pixel intensity of the red and green colors.

\section{Conclusion}

We have demonstrated a large intensity enhancement in the two-photon fluorescence emission of HPTS dye molecules by silver nanospheres. The silver nanospheres and dye molecules were co-encapsulated inside a polyacrylamide hydrogel matrix of larger nanoparticles. Signal enhancement by a factor of 20 was observed with $60 \mathrm{~nm}$ silver nanosphere cores, when excited at a wavelength of $900 \mathrm{~nm}$. We also tested $10 \mathrm{~nm}$ silver nanosphere cores but observed an enhancement of only 2.2 times. In addition to the usual MEF effect, the large observed enhancement is attributed to the excitation of the dye molecules by the second harmonic of the excitation light generated from the surface of the silver nanoparticles, when irradiated with NIR light. The generated second harmonic signal depends on the hyperpolarizability of the silver nanoparticles, which has been shown to increase with particle size. This method can be employed for any dye having an absorption in the visible region. Potentially this technique can also be used to generate and deliver visible light at tissue depths that are beyond the visible-light penetration depth. Consequently, this could be highly important for in vivo, deep in tissue, multiphoton imaging, sensing, or light-activated therapy, such as photodynamic therapy.

The polymeric cladding prevents silver nanoparticle aggregation, and thus any corresponding complicating optical effects; the polymeric matrix also protects the silver and the dye from interacting with proteins and other potentially interfering cell components. The embedding nanoparticle not only shields the silver and the dye but also potentially enables a long plasma lifetime, even longer if used with a polyethylene glycol (PEG) coating. ${ }^{[32]}$ In addition it allows potential specific targeting to any type of cell by surface engineering, such as attaching suitable targeting moieties to the matrix. ${ }^{[32]}$

We applied this nanoparticle sensor to image the intracellular $\mathrm{pH}$ in MDA-MB-435 cancer cells. Intracellular measurements showed that most of these nanoparticles end up inside acidic endosomes and we obtained an average $\mathrm{pH}$ value of 6.5. Such nanosensors could further be used for work on live tissues in vivo.

The HRS-based technique enables one to benefit from all the advantages of multiphoton fluorescence, such as good penetration depth, highly localized excitation, low autofluorescence, and high signal-to-noise ratio, even though a significant part of the fluorescence originates from a single-photon absorption mechanism. Furthermore, the required multishell nanoparticle structure fits seamlessly with the recent development of smart nanoparticle-based biosensors, such as 

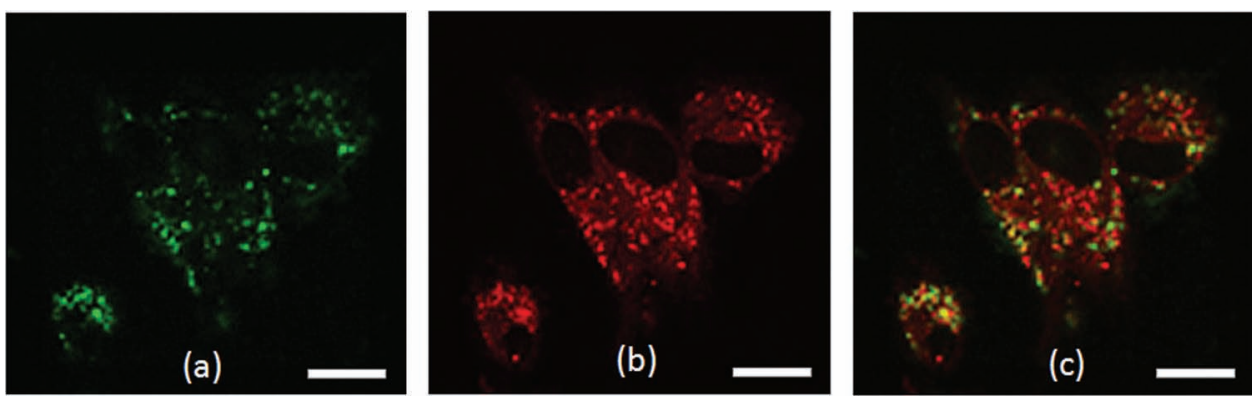

Figure 6. Fluorescence images from the cells. a) Fluorescence signal from the nanoparticles inside the MDA cells; b) fluorescence signal from the Lysotracker dye used to stain the acidic vesicles; and c) overlay of the fluorescence from nanoparticles (green) and the Lysotracker (red). Orange/ yellow shows the position of nanoparticles overlapping with acidic vesicles. Many of the nanoparticles are inside acidic vesicles; scale bar: $20 \mu \mathrm{m}$. This image was taken in the confocal mode.

nanoPEBBLES, as well as nanoactuators, such as targeted, theranostic, multifunctional, biodegradable, and bioeliminable nanoparticles for therapy, diagnostics, and surgical delineation. ${ }^{[32]}$

\section{Experimental Section}

Materials: Acrylamide, methylenebisacrylamide (MBA), dioctylsulfosuccinate (AOT), Brij 30, hexane, ammonium persulfate (APS), $N, N, N^{\prime}, N^{\prime}$-tetramethylethylenediamine (TEMED), $N$-(3dimethylaminopropyl)- $N^{\prime}$-ethylcarbodiimide hydrochloride (EDC) were all acquired from Sigma-Aldrich (St. Louis, MO). 3-(Aminopropyl)methacrylamide hydrochloride salt (APMA) was obtained from Polysciences Inc. (Warrington, PA). Ethanol (95\%) was acquired from Decon Laboratories, Inc. (King of Prussia, PA). HPTS was obtained from Invitrogen (Carlsbad, CA). The silver nanoparticles (Biopure) were obtained from Nanocomposix Inc. (San Diego, CA). All solutions were prepared in $18 \mathrm{M} \Omega$ water purified in a Barnstead 1 Thermolyne Nanopure II system. All the chemicals and materials were used as received.

Preparation of the Nanoparticles: The nanoparticles were prepared using a microemulsion and radical polymerization technique. The core was prepared by first adding HPTS $(19 \mathrm{mmol})$ to a solution $(1.6 \mathrm{~mL})$ of silver nanospheres (10 or $60 \mathrm{~nm})$ in citrate buffer $\left(1 \mathrm{mg} \mathrm{mL} \mathrm{L}^{-1}\right.$ concentration). The silver nanospheres had a citrate capping. Citrate is a small molecule and a relatively weak capping agent, so it may not prevent, but possibly limit, dye molecule adsorption. After the dye was adsorbed into the silver, the monomers acrylamide ( $8.6 \mathrm{mmol})$, APMA $(0.25 \mathrm{mmol})$, and MBA $(1.2 \mathrm{mmol})$ were added to it. This solution was then added to a hexane solution $(36 \mathrm{~mL})$ containing Brij $30(6.85 \mathrm{mmol})$ and AOT $(2.88 \mathrm{mmol})$. The two solutions were emulsified by stirring for 20 min under an inert atmosphere. The reaction was initiated by using TEMED $(0.54 \mathrm{mmol})$ and APS $(28 \mu \mathrm{mol})$, freshly prepared $(10 \%, \mathrm{w} / \mathrm{v})$ in water. The solution was further stirred for $2 \mathrm{~h}$ under an inert atmosphere to complete the polymerization. Hexane was removed by rotary evaporation using Rotavapor-R apparatus (Brinkmann Instruments), and then the residue was suspended in ethanol. The surfactants and excess dye were removed by washing the particles five times with ethanol and five times with water for over 2 days in an Amicon ultrafiltration cell (Millipore Corp., Bedford, MA), using a filter (300 kDa), and then freezedrying with a $5 \mathrm{~L}$ ModulyoD freeze dryer (ThermoFisher Scientific).
The rhodamine-containing nanoparticles were prepared by post-loading the rhodamine dye into blank polyacrylamide nanoparticles containing a silver core. Rhodamine is extremely soluble in ethanol and leached out of the nanoparticles while washing in ethanol. Therefore we post-loaded the dye into the nanoparticle by stirring it in an aqueous solution containing blank nanoparticles overnight. The nanoparticles were then washed thoroughly in water a few times to remove any extra dye and freeze dried.

Particle Size and Zeta Potential: The particles were diluted in water at a concentration of $1 \mathrm{mg} \mathrm{mL} \mathrm{m}^{-1}$ and the particle size distribution in aqueous solution and the zeta potential were measured by dynamic light scattering (DLS), using a Beckman-Coulter Delsa Nano C zeta potential/submicron size analyzer.

Silver Encapsulation: ICP Spectroscopy: The amount of silver encapsulated was determined by inductively coupled plasma (ICP)-optical emission spectroscopy using a Perkin-Elmer Optima 2000 DV machine. The nanoparticle sample concentration was $2 \mathrm{mg} \mathrm{mL}^{-1}$ in water.

$X$-Ray Diffraction: Powder XRD experiments were performed by using a Bruker D8 Advance powder X-ray diffractometer.

Dye Concentration: The amount of dye encapsulated in the nanoparticles was determined by comparing the fluorescence and absorbance from a known concentration of the nanoparticles in water to the fluorescence signals obtained from a set of known concentration of dye and silver nanoparticles in water. We first determined the amount of silver nanospheres present in the nanoparticle solution by performing ICP analysis. Calibration samples containing the same amount of free silver nanospheres were prepared while varying the concentration of the dye. We constructed the calibration curve by looking at the absorbance (and fluorescence) using the (free silver + dye) calibration solution and then comparing it with the absorbance/fluorescence of the nanoparticle solution. This was a bulk measurement and was quite accurate. The fluorescence measurements were performed in a FluoroMax-3 spectrofluorometer (JobinYvon-Spex).

Leaching: The nanoparticle solution was diluted in water at a concentration of $5 \mathrm{mg} \mathrm{mL}^{-1}$ and kept for $48 \mathrm{~h}$ under constant stirring. It was then centrifuged at $4000 \mathrm{rpm}$. The filtrate was collected and the concentration of the dye was determined by comparing the absorbance of the filtrate with the absorbance of a known concentration of dye. The absorption studies were carried out using a Shimadzu UV/Vis spectrometer. 
Calibration and Optical Characterization: The nanoparticles were dissolved in buffers of different $\mathrm{pH}$ at a concentration of $6.5 \mathrm{mg} \mathrm{mL}^{-1}$. The calibration was performed using a widely tunable femtosecond Ti:sapphire (Mai-Tai, Spectra Physics) laser. The laser had a pulse width of less than $100 \mathrm{fs}$ and operated at a frequency of $80 \mathrm{MHz}$. The central wavelength could be tuned from 690 to $950 \mathrm{~nm}$. The light was focused onto the sample using a 10× objective. Two excitation wavelengths, 900 and $740 \mathrm{~nm}$, were used and the fluorescence signal between 480 and $580 \mathrm{~nm}$ at each excitation wavelength was collected through the same objective and passed through a tunable acoustic filter before being collected using a photomultiplier tube (PMT). The ratio of the two fluorescence intensities was used for calibration. The enhancement was measured by taking the ratio of the fluorescence signal from the silver and dye encapsulated nanoparticle and an equal concentration of free dye by exciting them between 740 and $920 \mathrm{~nm}$. TEM was performed by using nanoparticle solutions $\left(0.01 \mathrm{mg} \mathrm{mL}^{-1}\right)$ and the negative staining of the polyacrylamide was achieved with uranyl acetate. The TEM was performed using a Philips CM-100 TEM microscope.

Cell Culture Procedures: MDA-MB-435 was chosen for intracellular sensing. MDA cells were cultivated in a Roswell Park Memorial Institute medium (RPMI-1640), supplemented with 10\% heatinactivated fetal bovine serum (Hi-FBS). The cells were plated on an eight-well chambered cover glass (Nunc. Lab-Tek) and grown for a few days before incubation with nanoparticles.

Cellular Uptake Procedures: The cells were incubated with nanoparticles of $1 \mathrm{mg} \mathrm{mL}^{-1}$ final concentration for $3 \mathrm{~h}$. After incubation, unbound nanoparticles were removed by gentle rinsing with fresh cell medium three times. Then the cells were treated with a lysosomal staining probe, Lysotracker Red DNB-99 for $10 \mathrm{~min}$. The excess Lysotracker probe was removed by washing with colorless Dulbecco's modified Eagle's medium (DMEM) one more time.

Fluorescence Microscopy: The two-photon and single-photon images of the cells loaded with nanoparticles were taken using a Leica confocal microscope (SP-5X), located at the Microscopy and Image Analysis Laboratory of the University of Michigan. The single-photon images were taken with the diode laser at 405 and $450 \mathrm{~nm}$, and the fluorescence emission was detected at $510 \mathrm{~nm}$. The single-photon images were acquired in the confocal mode with a slit width of 1 Airy unit. The two-photon images were taken by exciting the nanoparticles with 740 and $900 \mathrm{~nm}$ laser lines from the femtosecond Ti:sapphire laser, and the fluorescence emission was collected around $510 \mathrm{~nm}$. The two-photon images were acquired using either a $10 \times$ or $20 \times$ objective, whereas the single-photon images were acquired using a $40 \times$ or $60 \times$ oil-immersion objective. PMTs were used to collect the fluorescence in both cases.

MTT Assay for Toxicity Testing: Cells were cultivated on 96-well plates. For each treatment with nanoparticles and for a control (cells without any treatment), a total of 12 wells were used for reliable measurements. The nanoparticles containing silver and dye were added to cell medium to make the nanoparticle concentration $1 \mathrm{mg} \mathrm{mL} \mathrm{m}^{-1}$. For comparison, blank polyacrylamide nanoparticles were also added to cell medium at a similar concentration of $1 \mathrm{mg} \mathrm{mL} \mathrm{m}^{-1}$. After $4 \mathrm{~h}$ of incubation, the treated cells were washed three times with fresh cell medium to remove any unbound nanoparticles. A 3-(4,5-dimethylthiazol-2-yl)-2,5-diphenyltetrazolium bromide (MTT) solution (100 $\mu \mathrm{L}, 2.5 \mathrm{mg} \mathrm{mL}^{-1}$ in PBS) was added to each treated well and control. The cells were incubated for $4 \mathrm{~h}$ at $37^{\circ} \mathrm{C}$, the cell medium was removed, and dimethyl sulfoxide (DMSO, $100 \mu \mathrm{L}$ ) was added to solubilize the water-insoluble formazan that was formed by reduction of the MTT agent by live cell enzymes. The cell viability was determined by measuring the light absorbance of each well at a wavelength of $550 \mathrm{~nm}$ and comparing the results with those from the controls.

\section{Acknowledgements}

This work was supported in part by NSF grant no. NSF-DMR 0455330 (R.K.). We thank Dr. Hoe Jin Hah for his help and useful discussions on nanosensor synthesis, Dr. Antek Wong-Foyfor help during XRD measurements, Teppei Shirakura for help during nanoparticle calibration, Jacquelyn Priestley for help during synthesis of rhodamine nanoparticles, and Leshern Karamchand for useful discussions about the uptake of nanoparticles in cells. We also thank Chris Edwards, Sasha Meshinchi, and Dotty Sorenson at the Microscopy and Image Analysis Laboratory, the University of Michigan, for their technical support in confocal imaging and TEM imaging. The authors also thank Paul Lennon and James Windak at the Chemistry Instrument Shop for their technical support while using the ICP machine and the UV/Vis spectrometer.

[1] W. Denk, J. Strickler, W. Webb, Science 1990, 248, 73-76.

[2] F. Helmchen, W. Denk, Nat. Methods 2005, 2, 932-940.

[3] G. S. He, P. P. Markowicz, T. C. Lin, P. N. Prasad, Nature 2002, 415, 767-770.

[4] I. Cohanoschi, F. E. Hernandez, J. Phys. Chem. B 2005, 109, 14506-14512.

[5] H. Kano, S. Kawata, Opt. Lett. 1996, 21, 1848-1850.

[6] Y. Shen, J. Swiatkiewicz, T. C. Lin, P. Markowicz, P. N. Prasad, J. Phys. Chem. B 2002, 106, 4040-4042.

[7] I. Gryczynski, J. Malicka, Y. Shen, Z. Gryczynski, J. R. Lakowicz, J. Phys. Chem. B 2002, 106, 2191-2195.

[8] J. Lukomska, I. Gryczynski, J. Malicka, S. Makowiec, J. R. Lakowicz, Z. Gryczynski, Biochem. Biophys. Res. Commun. 2005, 328, 78-84.

[9] A. M. Glass, A. Wokaun, J. P. Heritage, J. G. Bergman, P. F. Liao, D. H. Olson, Phys. Rev. B 1981, 24, 4906-4909.

[10] R. He, Y. Su, K. Cho, C. Lin, N. Chang, C. Chang, S. Chen, Opt. Express 2009, 17, 5987-5997.

[11] W. Wenseleers, F. Stellacci, T. Meyer-Friedrichsen, T. Mangel, C. A. Bauer, S. J. K. Pond, S. R. Marder, J. W. Perry, J. Phys. Chem. B 2002, 106, 6853-6863.

[12] E. J. Sánchez, L. Novotny, X. S. Xie, Phys. Rev. Lett. 1999, 82, 4014-4017.

[13] L. Novotny, R. X. Bian, X. S. Xie, Phys. Rev. Lett. 1997, 79, 645-648.

[14] E. J. McLaurin, A. B. Greytak, M. G. Bawendi, D. G. Nocera, J. Am. Chem. Soc. 2009, 131, 12994-13001.

[15] P. Anger, P. Bharadwaj, L. Novotny, Phys.Rev. Lett. 2006, 96, 113002.

[16] K. Aslan, I. Gryczynski, J. Malicka, E. Matveeva, J. Lakowicz, C. D. Geddes, Curr. Opin. Biotechnol. 2005, 16, 55-62.

[17] C. Lin, K. Chiu, C. Chang, S. Chang, T. Guo, S. Chen, Opt. Express 2010, 18, 12807-12817.

[18] E. C. Hao, G. C. Schatz, R. C. Johnson, J. T. Hupp, J. Chem. Phys. 2002, 117, 5963-5966. 
[19] P. C. Ray, Chem. Rev. 2010, 110, 5332-5365.

[20] F. W. Vance, B. I. Lemon, J. T. Hupp, J. Phys. Chem. B 1998, 102, 10091-10093.

[21] M. Chandra, A. M. Dowgiallo, K. L. Knappenberger Jr., J. Phys. Chem. C 2010, 114, 19971-19978.

[22] T. Itoh, Y. Ozaki, H. Yoshikawa, T. Ihama, H. Masuhara, Appl. Phys. Lett. 2006, 88, 084102.

[23] R. C. Johnson, J. Li, J. T. Hupp, G. C. Schatz, Chem. Phys. Lett. 2002, 356, 534-540.

[24] A. Neely, C. Perry, B. Varisli, A. K. Singh, T. Arbneshi, D. Senapati, J. R. Kalluri, P. C. Ray, ACS Nano 2009, 3, 2834-2840.

[25] A. K. Singh, D. Senapati, S. Wang, J. Griffin, A. Neely, P. Candice, K. M. Naylor, B. Varisli, J. R. Kalluri, P. C. Ray, ACS Nano 2009, 3, 1906-1912.

[26] J. Griffin, A. K. Singh, D. Senapati, E. Lee, K. Gaylor, J. Jones-Boone, P. C. Ray, Small 2009, 5, 839-845.

[27] K. Aslan, M. Wu, J. R. Lakowicz, C. D. Geddes, J. Am. Chem. Soc. 2007, 129, 1524-1525.

[28] K. Aslan, M. Wu, J. R. Lakowicz, C. D. Geddes, J. Fluoresc. 2007, $17,127-131$
[29] H. A. Clark, P. J. Campagnola, J. P. Wuskell, A. Lewis, L. M. Loew, J. Am. Chem. Soc. 2000, 122, 10234-10235.

[30] P. J. Campagnola, H. A. Clark, W. A. Mohler, A. Lewis, L. M. Loew, J. Biomed. Opt. 2001, 6, 277-286.

[31] H. A. Clark, M. Hoyer, M. A. Philbert, R. Kopelman, Anal. Chem. 1999, 71, 4831-4836.

[32] Y. E. Koo Lee, R. Smith, R. Kopelman, Annu. Rev. Anal. Chem. 2009, 2, 57-76.

[33] Y. E. Koo Lee, E. E. Ulbrich, G. Kim, H. Hah, C. Strollo, W. Fan, R. Gurjar, S. M. Koo, R. Kopelman, Anal. Chem. 2010, 82, 8446-8455.

[34] A. Ray, Y. E. Koo Lee, T. Epstein, G. Kim, R. Kopelman, Analyst 2011, 136, 3616-3622.

[35] Y. Zhang, A. Dragan, C. D. Geddes, J. Phys. Chem. C 2009, 113, 12095-12100.

[36] M. R. Gartia, A. Hsiao, M. Sivaguru, Y. Chen, G. L. Liu, Nanotechnology 2011, 22, 365203.

Received: December 16, 2011

Revised: January 17, 2012

Published online: April 20, 2012 